\title{
High-level activities of daily living and disease-specific mortality during a I2-year follow-up of an octogenarian population
}

This article was published in the following Dove Press journal:

Clinical Interventions in Aging

14 June 2013

Number of times this article has been viewed

\author{
Yutaka Takata' \\ Toshihiro Ansai ${ }^{2}$ \\ Inho Soh ${ }^{2}$ \\ Shuji Awano 2 \\ Ikuo Nakamichi' \\ Sumio Akifusa ${ }^{3}$ \\ Kenichi Goto' \\ Akihiro Yoshida ${ }^{2}$ \\ Hiroki Fujii' \\ Ritsuko Fujisawa' \\ Kazuo Sonoki ${ }^{3}$ \\ 'Division of General Internal \\ Medicine, ${ }^{2}$ Division of Community \\ Oral Health Science, ${ }^{3}$ Department of \\ Oral Health and Environment, School \\ of Oral Health Science, Kyushu Dental \\ University, Kitakyushu, Japan
}

Correspondence: Yutaka Takata

Division of General Internal Medicine,

Kyushu Dental University,

Manazuru 2-6-I, Kokurakita-ku,

Kitakyushu 803-8580, Japan

Tel $+8 \mid 93582$ II3I ext 20 I I

Fax +8I 935820592

Email yutaka@kyu-dent.ac.jp
Background: Little is known about the relationship between disease-specific mortality and high-level activities of daily living in the elderly. We examined whether mortality is associated with high-level activities of daily living in an octogenarian population.

Methods: We conducted a population-based cross-sectional and prospective cohort study in 693 older persons aged 80 years and living in Japan's Fukuoka Prefecture. We then evaluated the association between 12-year disease-specific mortality and high-level functional capacity as measured by the Tokyo Metropolitan Institute of Gerontology Index of Competence, which is a standardized multidimensional 13-item instrument; items 1 through 5 are classified as instrumental self-maintenance activity, items 6 through 9 as intellectual activity, items 10 through 13 as social roles activity, and all 13 items together yield total functional capacity.

Results: By the 12-year follow-up of the 693 participants, 413 had died, 242 survived, and 38 were unable to be located. Of the 413 who died, 105 died of cardiovascular disease, 73 of respiratory tract disease, 71 of cancer, and 39 of senility. Of the other 125 deaths, 59 were due to other diseases, and the cause of death for 66 participants is not known. The hazard ratio (HR) for all-cause mortality, adjusted for confounding factors with multivariate Cox analyses, fell by $6 \%$ (HR $0.937,95 \%$ confidence interval [CI] $0.899-0.978, P=0.003$ ) with each one-point increase in participants' scores on the Tokyo Metropolitan Institute of Gerontology Index of total functional capacity. With one-point increases in instrumental self-maintenance activity and in intellectual activity, the HRs for all-cause mortality decreased by $14 \%$ (HR $0.856,95 \%$ CI $0.787-0.930, P=0.000$ ) and $12 \%$ (HR $0.884,95 \%$ CI $0.794-0.983, P=0.023$ ), respectively. Respiratory mortality with HR adjustment fell by $11 \%$ (HR $0.887,95 \%$ CI $0.804-0.978$, $P=0.016)$ and $24 \%$ (HR $0.760,95 \%$ CI $0.627-0.922, P=0.005)$ with one-point increases in the scores of total functional capacity and instrumental self-maintenance activity, respectively. Similarly, mortality due to senility fell by $16 \%$ (HR $0.838,95 \%$ CI $0.743-0.946, P=0.004$ ), $29 \%$ (HR $0.707,95 \%$ CI $0.564-0.886, P=0.003$ ), and $29 \%$ (HR $0.710,95 \%$ CI $0.522-0.966$, $P=0.029)$ with one-point increases in the scores of total functional capacity, instrumental selfmaintenance activity, and intellectual activity, respectively.

Conclusion: These findings suggest that high-level activities of daily living may be an independent predictor of mortality due to all causes, respiratory disease and senility in older persons. Keywords: older persons, community-dwelling, mortality, respiratory death, instrumental ADL

\section{Introduction}

Activities of daily living (ADL) can be divided into basic ADL and ADL that are high-level. Basic ADL include eating, bathing, dressing, being mobile, toileting, and maintaining urinary control. ${ }^{1}$ Examples of instrumental or high-level ADL are shopping; preparing meals; housekeeping; doing laundry, going out to nearby places; and 
managing transportation, medication, and money. ${ }^{2}$ High-level ADL can be assessed by the Tokyo Metropolitan Institute of Gerontology (TMIG) Index of Competence., ${ }^{3,4}$

Earlier studies showed that the ADL of walking, standing, sitting, going out, bathing, eating, toileting, and dressing, with or without assistance, are related to mortality in community-dwelling older Japanese persons aged at least 65 years. ${ }^{5,6}$ A higher impairment level according to minimum dataset subscale ADL levels was an independent predictor of shorter survival in persons aged 65 years or over. ${ }^{7}$ Difficulty with two, three, or more instrumental ADL (tasks essential to home management and independent living) was associated with higher mortality in adults aged 65 years or older. ${ }^{8}$ We also found an association between ADL dependency and disease-specific mortality in an older population. ${ }^{9}$ In another investigation, greater risk of incident dementia was found in an older, unhealthy, religious population, mean age 73.0 years, than in the other behavioral groups. ${ }^{10}$ In a nationally representative survey of older adults in the United States, which included 31,568 community dwelling adults aged 65 and over, the prevalence of multiple chronic disease was increasing, but the level of impairment and disability remained stable. ${ }^{11}$

Although total mortality is likely to be higher in older individuals with impaired ADL, little is known about the relationship between disease-specific mortality and highlevel ADL in older persons. Therefore, we examined whether 12-year disease-specific mortality is more closely associated with high-level ADL than with basic ADL in a communitydwelling population of octogenarians.

\section{Methods}

In 1998, a total of 1282 individuals residing in three cities (Buzen, Yukuhashi, Munakata), four towns (Katsuyama, Tikujo, Toyotsu, Kanda), one village (Shinyoshitomi), and one ward (Tobata of Kikakyushu City) within Japan's Fukuoka Prefecture were selected for this study; all individuals were 80 years old. The nine locations were selected randomly from among urban, suburban, and rural communities with the goal of achieving a balance of living environments including sociodemographic backgrounds, dietary habits, health behaviors, and medical treatment. There were no baseline inclusion and exclusion criteria for the study. Of the 1282 individuals, 693 (54.1\%) (276 males and 417 females) agreed to participate, and each participant underwent both a high-level ADL assessment and a laboratory blood examination. The 693 participants were followed-up 12 years after the baseline examination. Confirmation of whether the individual was living or had died was obtained by interviewing the family via a telephone call or home visit. We also asked the patient's family about the cause of death, and then coded it according to the International Classification of Diseases (ICD-10, World Health Organization). Thirty-eight participants (5.5\%) were lost over the 12 years and were unable to be located for follow-up. The study was conducted according to the principles expressed in the Declaration of Helsinki and was approved by the Human Investigations Committee of Kyushu Dental College. Informed consent for study participation was obtained from all participants.

The TMIG Index of Competence, a standardized multidimensional 13-item instrument developed by the TMIG group, was used to measure the participants' high-level ADL at the start of this study. ${ }^{3}$ Items 1 through 5 are classified as instrumental self-maintenance activity, items 6 through 9 as intellectual activity, items 10 through 13 as social roles activity, and all items together, 1 through 13, as total functional capacity. The response to each item was simply "yes" or "no" and was scored 1 for yes or 0 for no. The total score was the sum of the number of items answered yes; a high score indicated high-level functional capacity. A score for each subscale of this index was also used to measure the capacity levels of instrumental self-maintenance, intellectual activity, and social roles. Points for TMIG subscores were based on the tertile number.

All data are reported as medians (range). Differences in mean values between groups were assessed by unpaired $t$-test or analysis of variance. Categorical variables were compared using the $\chi^{2}$ test. Associations between high-level ADL and 12-year mortality were assessed using the multivariate Cox proportional hazards regression model. The TMIG index score, gender, smoking, and body mass index (BMI) as well as levels of albumin, total serum cholesterol, and glucose were fit as continuous variables, and these variables were also used as confounding factors. Comparisons of the survival rates among groups according to high-level ADL were assessed by the Kaplan-Meier method, followed by a log-rank test to assess the significance between survival curves. All statistical analyses were performed using SPSS 16.0 (SPSS Japan, Inc, Tokyo, Japan). Results were considered significant at $P<0.05$.

\section{Results}

During the 12-year period from April 1998 to March 2010, of the 693 individuals who participated in the present study, 413 died, 242 survived, and 38 were lost by the time of followup. The follow-up rate was $94.5 \%$, and the total mortality 
was $63.1 \%$. Of the 413 participants who died, 105 died due to cardiovascular disease, 73 to respiratory tract disease, 71 to cancer, 39 to senility, 18 to digestive system disease, 14 to exogenous death, seven to neurological disease, six to urinary tract diseases, and 14 to other diseases; the cause of death for the remaining 66 participants was not known. Death due to senility was usually diagnosed when the patient had poor ADL and died without a clear diagnosis.

Deaths due to cardiovascular disease included 37 heart failures, 23 brain infarctions, 15 myocardial infarctions, nine aortic aneurysms, three cases of brain bleeding, three cases of ischemic heart disease, two cases of hypertension, one stroke, one pulmonary infarction, one case of sick sinus syndrome, one case of subdural bleeding, one case of subarachnoidal bleeding, one carotid artery aneurysm, one case of angina, one cardiac rupture, and five deaths for which the details were unknown.

The 73 deaths due to respiratory tract diseases included 59 cases of pneumonia, eight respiratory failures, one case of bronchial asthma, one case of emphysema, one case of chronic bronchitis, one case of chronic obstructive lung disease, and two deaths for which the details were unknown.

The 71 cancer cases included 16 cases of lung cancer, 12 cases of gastric cancer, ten cases of hepatic cancer, six cases of colon cancer, five cases of urinary tract cancer, four cases of pancreatic cancer, four cases of uterine cancer, two cases of bile duct cancer, one case of esophageal cancer, one case of ovarian cancer, one case of leukemia, one case of gallbladder cancer, one case of multiple myeloma, one case of laryngeal cancer, one case of peritoneal cancer, one chondrosarcoma, one malignant lymphoma, one malignant mesothelioma, and two deaths for which the details were unknown.

The baseline characteristics of the participants who survived the 12-year follow-up period and those who died are shown in Table 1. Males and smokers were more likely to die compared to females and nonsmokers. The BMI, serum concentration of total cholesterol, and serum level of albumin were higher in the group of survivors than in the nonsurvivors, whereas the serum level of glucose was lower in the survivors.

Of the 693 individuals who participated in this study, 651 were classified by their TMIG Index of Competence scores as being high-level, and the other 42 participants were not classified. No significant difference was found in total mortality between these two groups of participants $(62.2 \%$ versus $75.6 \%, P=0.085$ ). The participants' total functional capacity scores were divided into a high-score group (scoring 12 or 13; $\mathrm{n}=213$ ), middle-score group (scoring 10 or $11 ; \mathrm{n}=219$ ), and low-score group (scoring 1 to $9 ; n=219$ ). Similarly, the participants' instrumental self-maintenance activity scores were divided into a high-score group (scoring $5 ; \mathrm{n}=362$ ), middle score (scoring $4 ; \mathrm{n}=147$ ), and low score (scoring 0 to $3 ; \mathrm{n}=142$ ). The intellectual activity scores were divided into a high-score group (scoring $4 ; \mathrm{n}=300$ ), middle-score group (scoring $3 ; \mathrm{n}=196$ ), and low-score group (scoring 0 to $2 ; \mathrm{n}=156$ ). The social roles activity scores were divided into a high-score group (scoring $4 ; \mathrm{n}=214$ ), middle-score group (scoring $3 ; \mathrm{n}=205$ ), and low-score group (scoring 0 to $2 ; \mathrm{n}=234$ ). Since there was one individual who participated only with the intellectual activity scoring, the number of participants for the total functional capacity was 651, and that for the intellectual activity was 652. In addition, two subjects only participated with social roles activity, so the number for social role activity scores was 653 .

All-cause mortality was most prevalent in the total functional capacity low-score group, more prevalent in the middle-score group, and least prevalent in the high-score group (Table 2). Similarly, there was a slight difference in mortality due to respiratory disease among the three total functional capacity groups; lowest in the high-score, lower in the middle-score, and high in the low-score group. No significant differences were found in mortality due to cardiovascular disease, cancer, or senility among the total functional capacity groups. The ADL-mortality associations were assessed separately for the rural-dwelling group and the city-dwelling group. There were 141 older people in the city group, and 208 older people in the rural group. All-cause mortality was higher in the total functional capacity low-score group and

Table I Baseline characteristics of the participants who survived during the 12-year follow-up and those who died

\begin{tabular}{|c|c|c|c|}
\hline & $\begin{array}{l}\text { Living } \\
\mathrm{n}=\mathbf{2 4 2}\end{array}$ & $\begin{array}{l}\text { Deceased } \\
n=413\end{array}$ & $P$-value \\
\hline Men/women & $64 / 178$ & $203 / 210$ & 0.000 \\
\hline Smoking (yes/no) & $11 / 229$ & $72 / 334$ & 0.000 \\
\hline $\begin{array}{l}\text { Alcohol drinking } \\
\text { (yes/no) }\end{array}$ & $131 / 100$ & $221 / 181$ & 0.672 \\
\hline $\begin{array}{l}\text { Going to the hospital } \\
\text { regularly (yes/no) }\end{array}$ & $196 / 36$ & $334 / 66$ & 0.746 \\
\hline BMI $\left(\mathrm{kg} / \mathrm{m}^{2}\right)$ & $23.0(17.0-32.1)$ & $22.5(15.3-38.8)$ & 0.005 \\
\hline $\begin{array}{l}\text { Total cholesterol } \\
(\mathrm{mg} / \mathrm{dL})\end{array}$ & $212.0(14 \mid-318)$ & $197.0(91-329)$ & 0.000 \\
\hline Albumin (mg/dL) & $4.3(3.5-5.3)$ & $4.2(2.8-4.9)$ & 0.000 \\
\hline Blood sugar (mg/dL) & $105(11-578)$ & $107(60-456)$ & 0.005 \\
\hline $\begin{array}{l}\text { Systolic blood } \\
\text { pressure }(\mathrm{mmHg})\end{array}$ & $|5|(|02-22|)$ & $148(100-258)$ & 0.522 \\
\hline
\end{tabular}

Note: Data are shown as medians (range).

Abbreviation: BMI, body mass index. 
lower in high-score group $\left(66.7 \%\right.$ versus $40.0 \% ; \chi^{2}=8.267$, $P=0.016$ ) who resided in city areas, while no difference was found between total functional capacity low-score and highscore group $\left(74.0 \%\right.$ versus $\left.58.0 \% ; \chi^{2}=4.316, P=0.116\right)$ who resided in rural areas.

As shown in Table 2, in a pattern similar to that of the total functional capacity groups, both the all-cause mortality and the respiratory mortality were most prevalent in the instrumental self-maintenance activity low-score group and more prevalent in the middle-score group compared to those in the high-score group. Mortality due to cardiovascular disease, cancer, or senility did not differ significantly among the instrumental self-maintenance activity groups.

Regarding the intellectual activity groups (Table 2), there was no significant difference in any cause of mortality among the groups. In contrast, the all-cause mortality was highest in the social roles activity low-score group, lower in the middle-score group and lowest in the high-score group, whereas cancer mortality was highest in the high-score social

Table 2 Mortality due to all-causes, cardiovascular disease, respiratory disease, cancer, or senility for the subscales of the Tokyo Metropolitan Institute of Gerontology (TMIG) index of competence

\begin{tabular}{|c|c|c|c|c|}
\hline Mortality & $\begin{array}{l}\text { High } \\
\text { score }\end{array}$ & $\begin{array}{l}\text { Middle } \\
\text { score }\end{array}$ & $\begin{array}{l}\text { Low } \\
\text { score }\end{array}$ & $P$-value \\
\hline \multicolumn{5}{|c|}{ Total functional capacity } \\
\hline All-cause & $56.0 \%$ & $60.2 \%$ & $70.6 \%$ & 0.008 \\
\hline Cardiovascular & $13.0 \%$ & $17.5 \%$ & $17.6 \%$ & 0.349 \\
\hline Respiratory & $8.0 \%$ & $9.5 \%$ & $15.2 \%$ & 0.049 \\
\hline Cancer & $15.0 \%$ & $9.5 \%$ & $8.3 \%$ & 0.071 \\
\hline Senility & $4.5 \%$ & $4.7 \%$ & $7.4 \%$ & 0.376 \\
\hline Men & $42.4 \%$ & $38.9 \%$ & $41.4 \%$ & 0.763 \\
\hline \multicolumn{5}{|c|}{ Instrumental self-maintenance activity } \\
\hline All-cause & $54.9 \%$ & $66.4 \%$ & $76.1 \%$ & 0.000 \\
\hline Cardiovascular & $14.2 \%$ & $19.3 \%$ & $17.4 \%$ & 0.353 \\
\hline Respiratory & $8.6 \%$ & $9.3 \%$ & $18.1 \%$ & 0.008 \\
\hline Cancer & $11.0 \%$ & $12.1 \%$ & $9.4 \%$ & 0.765 \\
\hline Senility & $4.2 \%$ & $8.6 \%$ & $5.8 \%$ & 0.156 \\
\hline Men & $36.7 \%$ & $50.4 \%$ & $41.9 \%$ & 0.020 \\
\hline \multicolumn{5}{|c|}{ Intellectual activity } \\
\hline All-cause & $61.8 \%$ & $58.2 \%$ & $68.4 \%$ & 0.149 \\
\hline Cardiovascular & $17.9 \%$ & $12.5 \%$ & $17.1 \%$ & 0.283 \\
\hline Respiratory & $8.9 \%$ & $13.6 \%$ & $11.2 \%$ & 0.286 \\
\hline Cancer & $12.1 \%$ & $9.8 \%$ & $9.9 \%$ & 0.654 \\
\hline Senility & $4.3 \%$ & $4.9 \%$ & $9.2 \%$ & 0.092 \\
\hline Men & $45.5 \%$ & $40.0 \%$ & $32.9 \%$ & 0.038 \\
\hline \multicolumn{5}{|c|}{ Social roles activity } \\
\hline All-cause & $56.7 \%$ & $60.8 \%$ & $68.3 \%$ & 0.045 \\
\hline Cardiovascular & $14.4 \%$ & $13.1 \%$ & $20.1 \%$ & 0.110 \\
\hline Respiratory & $7.7 \%$ & $10.1 \%$ & $14.7 \%$ & 0.065 \\
\hline Cancer & $14.4 \%$ & $12.1 \%$ & $6.7 \%$ & 0.032 \\
\hline Senility & $4.6 \%$ & $5.0 \%$ & $6.7 \%$ & 0.613 \\
\hline Men & $39.0 \%$ & $38.2 \%$ & $45.0 \%$ & 0.295 \\
\hline
\end{tabular}

roles activity group, lower in the middle-score group and lowest in the low-score group (Table 2).

\section{Overall survival rates and survival}

With the Kaplan-Meier method followed by a log-rank test to assess the significance between survival curves, it was revealed that the overall survival rates during the 12-year follow-up period were significantly higher in the total functional capacity high-score group $\left(\chi^{2}=11.5, P=0.001\right)$ and middle-score group $\left(\chi^{2}=7.4, P=0.007\right)$ compared to the low-score group (Figure 1A). The overall survival rate was highest in the instrumental self-maintenance activity high-score group $\left(\chi^{2}=27.2, P=0.000\right)$, lower in the middle-score group $\left(\chi^{2}=7.2, P=0.007\right)$, and lowest in the low-score group (Figure 1B). The survival curves of both the high-score $\left(\chi^{2}=4.5, P=0.034\right)$ and middlescore groups $\left(\chi^{2}=5.0, P=0.026\right)$ of intellectual activity were significantly higher than those of the low-score group (Figure 1C). Similarly, the survival curve of the social roles activity high-score group was significantly higher than that of the low-score group $\left(\chi^{2}=6.0, P=0.014\right)$ (Figure 1D).

Cardiovascular mortality was lower in the high-score groups for total functional capacity $\left(\chi^{2}=4.9, P=0.027\right)$, instrumental self-maintenance activity $\left(\chi^{2}=4.3, P=0.038\right)$ and social roles activity $\left(\chi^{2}=4.4, P=0.037\right)$ compared to their respective low-score groups, while no association was found between intellectual activity and cardiovascular mortality. Similarly, mortality from respiratory disease was lowest in the high-score groups for total functional capacity, instrumental self-maintenance activity, and social roles activity $\left(\chi^{2}=8.8, P=0.003 ; \chi^{2}=16.1, P=0.000 ; \chi^{2}=7.4\right.$, $P=0.006)$, higher in the respective middle-score groups $\left(\chi^{2}=5.5, P=0.019 ; \chi^{2}=6.6, P=0.010 ; \chi^{2}=2.7, P=0.103\right)$, and highest in the respective low-score groups. Senility mortality was also associated with instrumental self-maintenance activity and intellectual activity, but was not associated with total functional capacity or social roles activity. There was no association between cancer mortality and any score groups for total functional capacity, instrumental self-maintenance activity, intellectual activity, or social roles activity.

Table 3 shows the results of our multivariate Cox analyses of mortality and the TMIG index scores for total functional capacity, instrumental self-maintenance activity, intellectual activity, and social roles activity, which were adjusted for gender, smoking, BMI, serum level of albumin, glucose, and total cholesterol. Since all participants were 80 years old at the start of the study, age was not included as a confounding factor. The hazard ratio (HR) for each score of the 


\section{A}

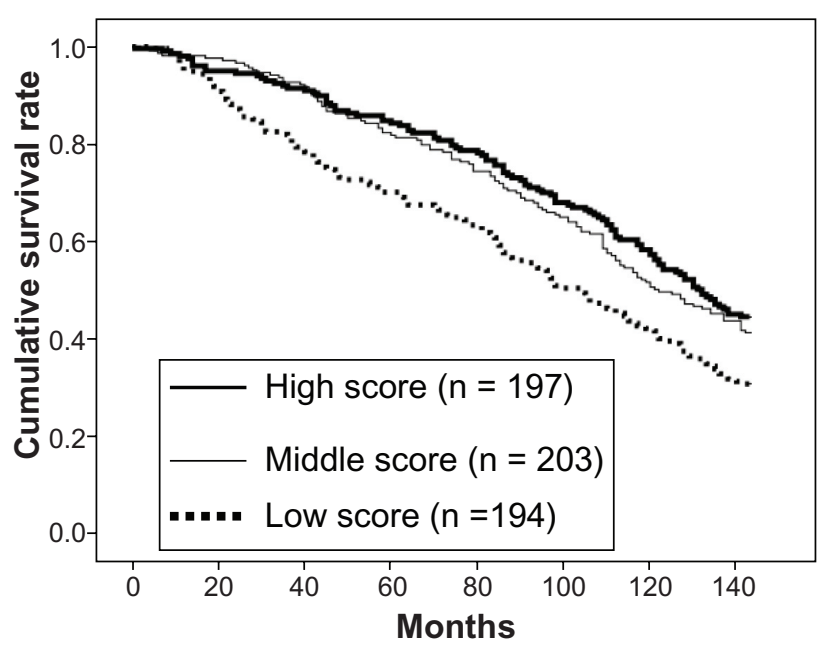

C

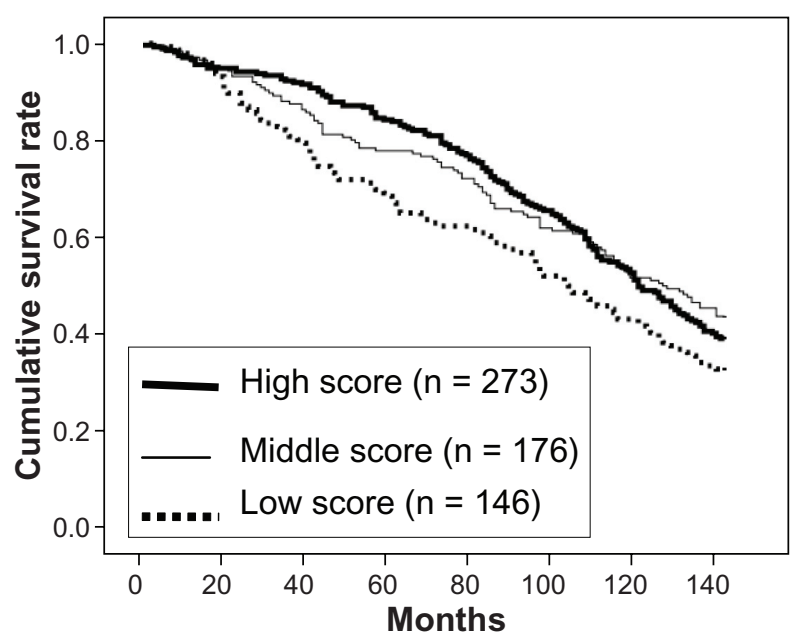

B

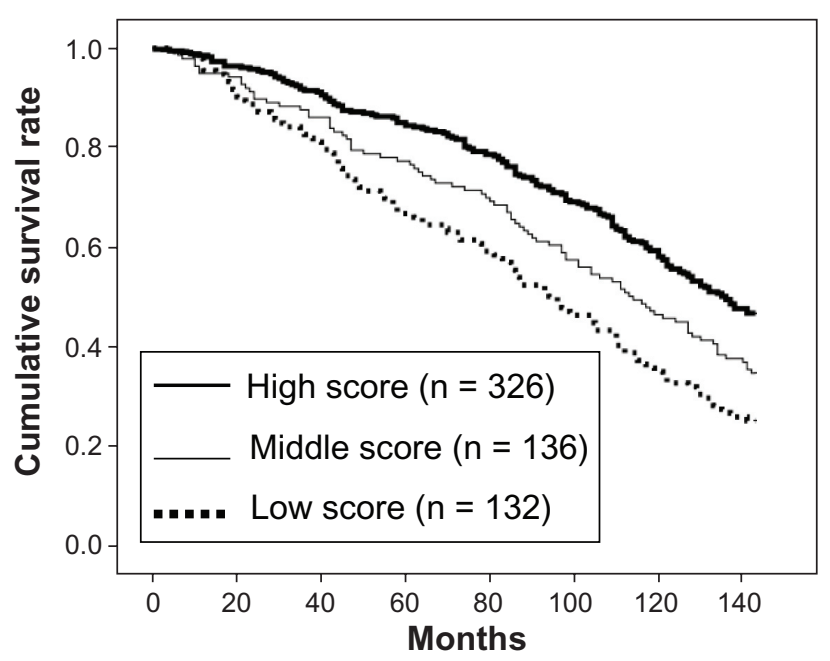

D

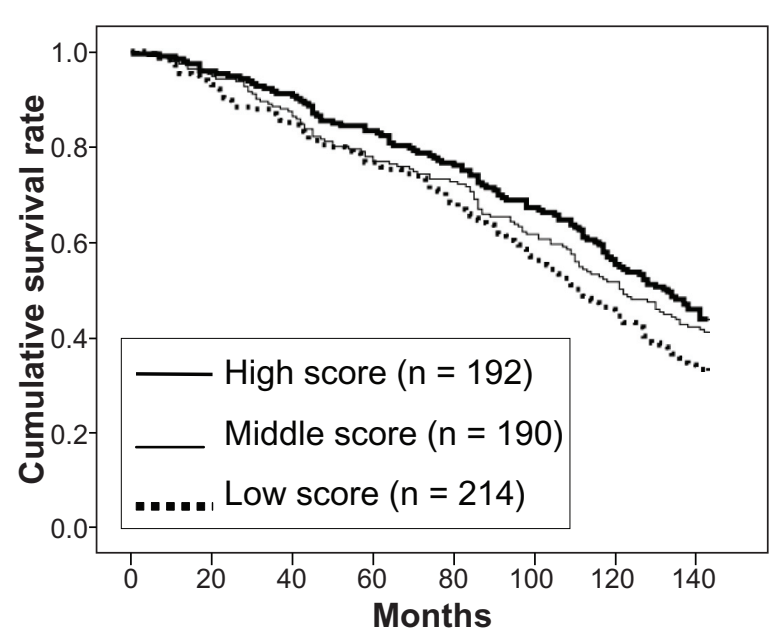

Figure I Overall survival curves during the 12-year follow-up period for the total functional capacity (A), instrumental self-maintenance activity (B), intellectual activity (C), and social roles activity (D).

TMIG index shows the risk of mortality associated with a one-point increase (continuous analysis) in score. The HR for all-cause mortality, adjusted for the above-mentioned confounding factors, fell by $6 \%$ with each one-point increase in total functional capacity (Table 3 ). Similarly, with onepoint increases in instrumental self-maintenance activity and in intellectual activity, the HR for all-cause mortality decreased by $14 \%$ and $12 \%$, respectively (Table 3 ), while no association was found between social role activity and total mortality (Table 3).

No association was found between cardiovascular mortality and the scores of total functional capacity, instrumental self-maintenance activity, intellectual activity, and social roles activity (Table 3 ). In contrast, cancer mortality rose by $40 \%$ with a one-point increase in the score of social roles activity (Table 3), and no association was found for cancer mortality with scores of total functional capacity, instrumental self-maintenance activity, and intellectual activity (Table 3).

Respiratory mortality adjusted for the same confounding factors fell by $11 \%$ and $24 \%$ with one-point increases in the scores for total functional capacity and instrumental selfmaintenance activity, respectively, but this mortality did not relate to the scores for intellectual activity and social roles activity (Table 3 ). Similarly, mortality due to senility fell by $16 \%, 29 \%$, and $29 \%$ with one-point increases in the scores for total functional capacity, instrumental self-maintenance activity, and intellectual activity, respectively, but there was no association between senility mortality and social roles activity (Table 3). 
Table 3 Multivariate Cox analyses of the association of gender, smoking, body mass index, serum level of albumin, glucose and total cholesterol with mortality of all-cause, cardiovascular disease, respiratory disease, cancer and senility

\begin{tabular}{|c|c|c|c|}
\hline Mortality & Hazard ratio & $95 \% \mathrm{Cl}$ & $P$-value \\
\hline \multicolumn{4}{|c|}{ Total functional capacity } \\
\hline All-cause & 0.937 & $0.899-0.978$ & 0.003 \\
\hline Cardiovascular disease & 0.945 & $0.870-1.026$ & 0.179 \\
\hline Respiratory disease & 0.887 & $0.804-0.978$ & 0.016 \\
\hline Cancer & 1.111 & $0.993-1.244$ & 0.066 \\
\hline Senility & 0.838 & $0.743-0.946$ & 0.004 \\
\hline \multicolumn{4}{|c|}{ Instrumental self-maintenance activity } \\
\hline All-cause & 0.856 & $0.787-0.930$ & 0.000 \\
\hline Cardiovascular disease & 0.916 & $0.773-1.087$ & 0.316 \\
\hline Respiratory disease & 0.760 & $0.627-0.922$ & 0.005 \\
\hline Cancer & $\mathrm{I} .155$ & $0.914-1.460$ & 0.228 \\
\hline Senility & 0.707 & $0.564-0.886$ & 0.003 \\
\hline \multicolumn{4}{|l|}{ Intellectual activity } \\
\hline All-cause & 0.884 & $0.794-0.983$ & 0.023 \\
\hline Cardiovascular disease & 0.921 & $0.750-1 .|3|$ & 0.432 \\
\hline Respiratory disease & 0.887 & $0.682-1.153$ & 0.370 \\
\hline Cancer & 1.021 & $0.785-1.327$ & 0.879 \\
\hline Senility & 0.710 & $0.522-0.966$ & 0.029 \\
\hline \multicolumn{4}{|l|}{ Social roles activity } \\
\hline All-cause & 0.965 & $0.879-1.059$ & 0.452 \\
\hline Cardiovascular disease & 0.911 & $0.764-1.086$ & 0.297 \\
\hline Respiratory disease & 0.830 & $0.668-1.032$ & 0.094 \\
\hline Cancer & $\mathrm{I} .398$ & I.088-I.797 & 0.009 \\
\hline Senility & 0.835 & $0.627-1.114$ & 0.220 \\
\hline
\end{tabular}

Abbreviation: $\mathrm{Cl}$, confidence interval.

\section{Discussion}

Our analyses revealed associations between 12-year mortality and high-level ADL scores measured in the octogenarian participants at baseline using a standardized multidimensional 13-item instrument developed by the TMIG group. High total functional capacity scores were related to decreases in all-cause mortality, respiratory disease, and senility, with adjustments for the confounding factors of gender; smoking; BMI; and serum levels of albumin, glucose, and total cholesterol. Similarly, increases in instrumental self-maintenance activity were related to decreases in mortality from all causes, respiratory disease, and senility. Intellectual activity was also related to decreases in both the all-cause and senility mortalities, although the significance of this relation was not high. Social roles activity was associated only with cancer mortality. Although there were other well-known ADL indexes, such as Barthel's ADL index, ${ }^{12}$ Katz's ADL score, ${ }^{13}$ and Lawton and Brody's instrumental ADL score, ${ }^{14}$ in 1998, we assessed ADL using the TMIG Index of Competence only. Therefore, we evaluated the association between mortalities and ADLs with TMIG Index of Competence.
These findings indicate that among 80 -year-olds, a decline in high-level ADL - especially instrumental self-maintenance activities such as using public transportation, shopping, preparing meals, arranging to pay bills, and handling one's own banking - was an independent predictor of increased risk of 12-year mortality from all causes, respiratory disease and senility. A decrease in the intellectual activity of filling out pension forms, reading newspapers, books and magazines, and being interested in news programs related to health was also an independent predictor of increased risk of mortality due to all causes and senility. Increased social roles activities such as interacting with friends, being asked for advice, visiting sick friends, and initiating conversation with young people were a probable independent predictor of increased risk of cancer mortality. Considering the $P$-value obtained in the Cox analyses (Table 3), Kaplan-Meier analyses (Figure 1) and $\chi^{2}$ tests (Table 2 ), the association with mortality was tighter for instrumental self-maintenance activity and total functional capacity than for intellectual activity and social roles activity, suggesting that instrumental self-maintenance activity and total functional capacity may be better predictors of the mortality of older individuals than intellectual activity and social roles activity.

However, our findings indicate that although the mortality from all causes, respiratory disease, and senility can be predicted by the level of instrumental self-maintenance activity, mortality due to cardiovascular disease and cancer cannot be predicted by high-level ADL scores including instrumental self-maintenance and intellectual and social roles. Similarly, we previously found that ADL dependency - ie, whether participants were independent in their everyday lives, such as being able to go out and use public transportation or going out into the neighborhood without assistance, versus needing assistance when living indoors and living on a bed in the daytime, needing assistance with toileting, eating meals, and changing clothes - was a predictor of mortality from all causes, respiratory disease and senility, but was not a predictor of mortality from cancer and cardiovascular disease. ${ }^{9}$ These findings suggest that not only ADL dependency, but also high-level ADL such as instrumental self-maintenance activity, are tightly associated with mortality due to all causes, respiratory disease, and senility.

In the present study, we found a positive association between cancer mortality and social roles activity, suggesting that individuals who are able to interact with friends, be asked for advice, visit sick friends, and initiate conversations with young people are more likely to die of cancer than individuals who are not able to perform these activities. The HR for 
cancer mortality with high-level ADL activity was greater than 1.0 , not only in social roles activity (1.398) but also in total functional capacity (1.111), instrumental self-maintenance activity (1.155), and intellectual activity (1.021), suggesting that individuals who have high-level ADL activity are more likely to die of cancer, and less likely to die of respiratory disease, senility, and cardiovascular disease. Increases in physical activity or physical fitness, however, are reported by several investigators to be associated with decreases in cancer mortality. ${ }^{15}$ In other studies, leisure-time activity was inversely related with all-cancer mortality in men aged 40-64 years ${ }^{16}$ and $40-69$ years. ${ }^{17}$ Similarly, physically active men at the average age of approximately 43 years ${ }^{18}$ or 54 years ${ }^{19}$ were at lower risk of death from cancer than sedentary men of similar ages. However, little is known about these associations in older populations (ie, over 80 years of age). Our participants were octogenarians and were followed from the age of 80 to 92. It seems plausible that very old individuals who do not die of respiratory disease, senility, or cardiovascular disease are likely to die of cancer because of their impaired immunity due to aging. However, further research is needed to elucidate this association.

Several studies have reported that cardiovascular disease mortality was increased in a dose-related manner relative to decreased physical activity. ${ }^{15-17,20}$ In the present study, no significant association was found between cardiovascular mortality and total functional capacity, instrumental selfmaintenance activity, intellectual activity, or social roles activity. However, the HR for death due to cardiovascular disease was consistently lower than 1.0 (0.945 for total functional capacity, 0.916 for instrumental self-maintenance activity, 0.921 for intellectual activity, 0.911 for social roles activity). Therefore, it seems likely that an inverse association between high-level functional capacity and cardiovascular disease mortality may exist, but it did not reach a significant level. This may be due to the small number of participants, or because the age of the participants was very high.

We found stronger associations between mortality due to respiratory disease or senility and total functional capacity or instrumental self-maintenance activity compared to those between mortality due to cardiovascular disease or cancer and total functional capacity or instrumental self-maintenance activity. Similarly, walking, as a physical activity, in men aged $40-64$ years $^{16}$ or $40-69$ years ${ }^{17}$ was inversely related to mortality due to respiratory disease in a previous study. Inverse associations between leisure-time physical activity and mortality were strongest for respiratory mortality compared with those for cancer or cardiovascular mortality in participants older than 35 years. ${ }^{21}$ Since the diagnosis of death due to senility was usually made in patients who had poor ADL and no clear diagnosis of induced death, the close association between senility-related mortality and total functional capacity or instrumental self-maintenance activity found in this study can be considered reasonable.

In the present study of an octogenarian population, the percent of subjects with a high-score (scoring 12 or 13) in total functional capacity was $32.4 \%$ (213/657), while in another study, the percent of Japanese individuals aged 65-83 years whose score was 13 points for total TMIG-index was $29.3 \%-63.1 \%,{ }^{22}$ indicating that the baseline rate for highscore ADL based on total TMIG-index was considerably low in our study. Relatively lower ADL levels may be attributed to the participants' age ( 80 years). During the 12-year follow-up, all-cause mortality was $63.2 \%(413 / 653)$ for octogenarians in the present study, $51.8 \%$ for an older Italian population aged 74.1 years, ${ }^{23}$ and $29.9 \%$ for Japanese women aged $67-89 .{ }^{24}$ Higher mortality in our study may be the result of older age or gender difference.

There were limitations in our study: the age of the subjects was limited to octogenarians, and the number of subjects was 693, which is considerably small. The race of our subjects was limited to Japanese only. Confirmation of whether the individual was living or deceased as well as the determination of the cause of death were both obtained only by asking the family via a telephone call or home visit. The present findings, however, still clearly suggest that high-level ADL may be an independent predictor of mortality due to all causes, respiratory disease, and senility in very old persons.

In conclusion, within an octogenarian population, we found associations between mortality rates from all causes, respiratory disease, and senility and the participants' total functional capacity or instrumental self-maintenance activities with adjustments for confounding factors. This suggests that high-level ADL, especially instrumental self-maintenance activities, may be independent predictors of mortality due to all causes, respiratory disease, and senility in very old persons. Therefore, it seems important, even in the very old, to improve functional capacity or instrumental self-maintenance activities in order to extend their lifespan.

\section{Acknowledgments}

This work was supported in part by Grants-in-Aid for Scientific Research (B) from Japan Ministry of Education, Culture, Sports, Science and Technology; 21390560 
(representative; Yutaka Takata) and 22390403 (representative; Toshihiro Ansai).

\section{Disclosure}

The authors report no conflicts of interest in this work.

\section{References}

1. Nishinaga M. Comprehensive geriatric assessment and team intervention. JMAJ. 2007;50(6):461-466.

2. Finlayson M, Mallinson T, Barbosa VM. Activities of daily living (ADL) and instrumental activities of daily living (IADL) items were stable over time in a longitudinal study on aging. J Clin Epidemiol. 2005;58(4): 338-349.

3. Koyano W, Shibata H, Nakazato K, Haga H, Suyama. Measurement of competence: reliability and validity of the TMIG index of competence. Arch Gerontol Geriatr. 1991;13(2):103-116.

4. Shibata H, Sugisawa H, Watanabe S. Functional capacity in Japanese living in the community. Geriatr Gerontol Int. 2001;1(1-2):8-13.

5. Matsubayashi K, Okumiya K, Osaki Y, Fujisawa M, Doi Y. Frailty in elderly Japanese. Lancet. 1999;353(9162):1445.

6. Konno K, Katsumata Y, Arai A, Tamashiro H. Functional status and active life expectancy among senior citizens in a small town in Japan. Arch Gerontol Geriatr. 2004;38(2):153-166.

7. Lee JS, Chau PP, Hui E, Chan F, Woo J. Survival prediction in nursing home residents using the minimum data set subscales: ADL self-performance hierarchy, cognitive performance and the changes in health, end-stage disease and symptoms and signs scales. Eur J Pubulic Health. 2009;19(3):308-312.

8. Fried LP, Kronmal RA, Newman AB, et al. Risk factors for 5-year mortality in older adults: the cardiovascular health study. JAMA. 1998;279(8):585-592.

9. Takata Y, Ansai T, Soh I, et al. Activities of daily living dependency and disease-specific mortality during 12-year follow-up in an 80-year-old population. Aging Clin Exp Res. 2013;25(2):193-201.

10. Norton MC, Dew J, Smith H, et al. Lifestyle behavior pattern is associated with different levels of risk for incident dementia and Alzheimer's disease: the Cache Country study. J Am Geriatr Soc. 2012;60(3): 405-412.

11. Hung WW, Ross JS, Boockvar KS, Siu AL. Recent trends in chronic disease, impairment and disability among older adults in the United States. BMC Geriatrics. 2011;11:47.
12. Wade DT, Collin C. The Barthel ADL index: a standard measure of physical disability? Int Disabil Stud. 1988;10(2):64-67.

13. Duarte YA, de Andrade CL, Lebrão ML. Katz index on elderly functionality evaluation. Rev Esc Enferm USP. 2007;41(2):317-325.

14. Lawton MP, Brody EM. Assessment of older people: self-maintaining and instrumental activities of daily living. Gerontologist. 1969;9(3): 179-186.

15. Blair SN, Cheng Y, Holder JS. Is physical activity or physical fitness more important in defining health benefits? Med Sci Sports Exerc. 2001;33(Suppl 6):S379-S399.

16. Davey Smith G, Shipley MJ, Batty GD, Morris JN, Marmot M. Physical activity and cause-specific mortality in the Whitehall study. Public Health. 2000;114(5):308-315.

17. Batty GD, Shipley MJ, Kivimaki M, Marmot M, Davey Smith G. Walking pace, leisure time physical activity, and resting heart rate in relation to disease-specific mortality in London: 40 years follow-up of the original Whitehall study. An update of our work with professor Jerry N Morris (1910-2009). Ann Epidemiol. 2010;20(9):661-669.

18. Kampert JB, Blair SN, Barlow CE, Kohl HW 3rd. Physical activity, physical fitness, and all-cause and cancer mortality: a prospective study of men and women. Ann Epidemiol. 1996;6(5):452-457.

19. Park MS, Chung SY, Chang Y, Kim K. Physical activity and physical fitness as predictors of all-cause mortality in Korean men. $J$ Korean Med Sci. 2009;24(1):13-19.

20. Erlichman J, Kerbey AL, James WP. Physical activity and its impact on health outcomes. Paper 1: the impact of physical activity on cardiovascular disease and all-cause mortality: an historical perspective. Obes Rev. 2002;3(4):257-271.

21. Lam TH, Ho SY, Hedley AJ, Mak KH, Leung GM. Leisure time physical activity and mortality in Hong Kong: case-control study of all adult deaths in 1998. Ann Epidemiol. 2004;14(6):391-398.

22. Fujiwara Y, Shinkai S, Kumagai S, et al. Changes in TMIG-index of competence by subscale in Japanese urban and rural community older populations: six years prospective study. Geriatr Gerontol Int. 2003;3(Suppl 1):S63-S68.

23. Testa G, Cacciatore F, Della-Morte D, et al. Role of permanent atrial fibrillation (AF) on long-term mortality in community-dwelling elderly people with and without chronic heart failure (CHF). Arch Gerontol Geriatr. 2012;55(1):91-95.

24. Suzuki T, Yoshida H. Low bone mineral density at femoral neck is a predictor of increased mortality in elderly Japanese women. Osteoporos Int. 2010;21(1):71-79.
Clinical Interventions in Aging

\section{Publish your work in this journal}

Clinical Interventions in Aging is an international, peer-reviewed journal focusing on evidence-based reports on the value or lack thereof of treatments intended to prevent or delay the onset of maladaptive correlates of aging in human beings. This journal is indexed on PubMed Central, MedLine, the American Chemical Society's 'Chemical Abstracts

\section{Dovepress}

Service' (CAS), Scopus and the Elsevier Bibliographic databases. The manuscript management system is completely online and includes a very quick and fair peer-review system, which is all easy to use. Visit $\mathrm{http}: / /$ www.dovepress.com/testimonials.php to read real quotes from published authors. 